\title{
REVIEW OF COMMERCIAL CELLS FOR SPACE APPLICATIONS
}

\author{
Rachel Buckle $^{(1)}$, Sam Roberts ${ }^{(1)}$ \\ (I) ABSL Space Products, F4 Culham Science Centre, Abingdon, UK.Email: rachel.buckle@absl.enersys.com
}

\begin{abstract}
Advances in Li-ion technology mean that manufacturers currently produce cells that have higher energy densities, higher rate capabilities, and longer quoted cycle lives than the currently qualified ABSL space cells. It is therefore possible that cells are available that could enable a lower mass/volume solution for a given application. ABSL carry out regular reviews of commercial off the shelf (COTS) cells, to determine whether any available cells are suitable for space applications, and offer superior performance to those currently qualified.
\end{abstract}

This paper describes the process that is followed to review and test commercial cells.

\section{INTRODUCTION}

ABSL has used the $18650 \mathrm{HC}(\mathrm{M})$ for space batteries since 1998; it was employed on the first ever Li-ion space battery for the PROBA-1 mission. Although the detailed performance and life-time characteristics of these cells are very well understood, advances in Li-ion technology mean that manufacturers currently produce cells that have double the energy density of this heritage cell. In addition, cells are available with the same energy density as the $\mathrm{HC}(\mathrm{M})$ but with considerably higher rate capabilities. Cells with longer cycle life have also been developed, although there is normally a trade-off in energy density such that a simple comparison cannot be made for end-of-life (EoL) performance.

To date, ABSL have qualified three Li-ion cells for space applications, these are:

- 18650HC(M). The heritage ABSL cell has a nominal capacity of $1.5 \mathrm{Ah}(133 \mathrm{Wh} / \mathrm{kg})$ and has been extensively characterised. The design was modified in 2009 to include a central mandrel; this was to ensure a clear vent path in the case of overpressure. The cell was completely re-qualified following this change.

- 18650HR. This cell was qualified specifically for a launch vehicle program and later adopted for MARES on the ISS. The cell has lower capacity than the $\mathrm{HC}(\mathrm{M})$ cell, but can deliver significantly higher currents.

- 18650NL. This cell provides a considerable increase in energy density compared to the $\mathrm{HC}(\mathrm{M})$ cell: $189 \mathrm{Wh} / \mathrm{kg}$ compared to $133 \mathrm{Wh} / \mathrm{kg}$.

COTS cells have been recently assessed to identify those that may provide a performance benefit compared to the currently qualified cells. Due to the long durations for full qualification, and the quantity of life test data required to predict the long term performance, these reviews are carried out regularly.

\section{REVIEW PROCESS}

The initial stage of any COTS assessment is to determine what cells are available on the market. During the last market survey, cells from over 40 companies were assessed. For this review, a commercially available database was used for the initial search [1]. The database was used to filter the available cells by chemistry and form factor; these were then ranked by performance requirements, e.g. energy density and cycle life.

Once the initial list of cells was acquired from the database, the cell specification sheets were obtained. The data from these sheets were then assessed in detail against the cell performance requirements described below.

\section{CELL REQUIREMENTS}

The cell requirements are based on three factors: energy density, cycle life, and rate capability. There are obviously other desirable qualities for a space cell, for example launch vibration tolerance and survival under vacuum, however data on these traits is not available for the majority of COTS cells.

\subsection{Energy Density}

Since the introduction of Li-ion space batteries in the late 90's, the technology has developed and become more accepted. This has led to the steady reduction in mass budgets and an increase in the energy and rate requirements.

Some projects have requirements that necessitate the EoL energy density to be around $150 \mathrm{Wh} / \mathrm{kg}$, with development projects demanding more. Obviously, the beginning-of-life (BoL) energy density needs to be greater than this; the actual value will depend on the fade. 
The other energy criterion that was used to assess cells is from an ESA project to develop a Li-ion cell, with a specific energy of $250 \mathrm{Wh} / \mathrm{kg}$ or greater [2].

\subsection{Cycle Life / Fade}

LEO missions are now commonly in excess of 11 years comprising $\sim 60,000$ cycles(without electric propulsion and significantly more with), with GEO missions commonly specified for $\geq 15$ years operation. The fade of any selected cells should allow the EoL energy density requirement (described above) to be achieved.

However for COTS cells, the only data commonly available is the retained capacity after 200-300 cycles between maximum and minimum voltage, rather than for more realistic lower depth of discharge (DoD) regimes. Therefore, this COTS cell assessment bases the cycle performance on a comparison with the ABSL $18650 \mathrm{HC}(\mathrm{M})$ cell. Measured ABSL data show that the HCM cells complete at least 1500 cycles at $100 \%$ DoD to $80 \%$ retained capacity.

\subsection{Rate Capability}

For missions that require SAR operation, the maximum discharge currents and system minimum voltage requirements for typical high energy COTS cells can be a limiting factor. This may be due to high cell resistance, the operation of safety devices, or the temperature rise.

The ability to discharge at rates $>2 \mathrm{C}$ with minimal impact to fade would therefore be considered an advantage.

\section{SURVEY OF MANUFACTURERS AND CELLS}

\subsection{Manufacturers}

As part of the market review, an assessment of the manufacturer, as well as that of the cell type, is required. In this phase of the project, the cell manufacturer was assessed based on historical relationships. For the manufacturer of each selected cell type, the following areas have been considered.

- Quality. Issues can include variability of cell capacity, fade, corrosion, seal leakage.

- Relationship. Responses to queries, open discussion on the end use of the cells, and the battery design.

- Flexibility/customisation. Possibility of custom cells or specific delivery conditions.

- Experience/heritage. Particularly important for Space applications, where customers are trying to minimise risk of battery causing spacecraft failure.

\subsection{Cells}

Data from the manufacturers' specification sheets were assessed against the cell requirements detailed in Section 3. In order to summarise the cell performance and allow comparison between manufacturers, the cell performance was scored according to the criteria in Table 1. The energy density and cycle life were considered the most important requirements, whereas the rate capability is less significant.

Table 1. Cell scoring system

\begin{tabular}{|c|l|}
\hline Requirement & \multicolumn{1}{|c|}{ Scoring } \\
\hline & $0=<130$ \\
Energy Density & $1=130-160$ \\
(Wh/kg) & $2=160-190$ \\
& $3=190-210$ \\
& $4=>210$ \\
\hline & $0=<299$ \\
Cycle Life & $1=300-399$ \\
(no. of cycles to & $2=400-599$ \\
80\% capacity) & $3=600-999$ \\
& $4=>1000$ \\
\hline Rate & $0=$ max discharge rate $\leq 2$ \\
Capability (C) & $1=$ max discharge rate $2-5$ \\
& $2=$ max discharge rate $>5$ \\
\hline
\end{tabular}

Following the review (Table 2), the cells could be described in three groups:

- Very high energy density: up to $260 \mathrm{Wh} / \mathrm{kg}$ (Types 5, 7,8).

- High cycle life (can complete $>1000 \quad 100 \%$ cycles with less than $20 \%$ fade). These cells typically have lower energy densities (Types 1 , $2,4)$.

- In between these two extremes (Types 3,6).

Table 2. Cell performance scores

\begin{tabular}{|c|c|c|c|c|}
\hline Cell & $\begin{array}{c}\text { Energy } \\
\text { Density }\end{array}$ & $\begin{array}{c}\text { Cycle } \\
\text { Life }\end{array}$ & $\begin{array}{c}\text { Rate } \\
\text { Capability }\end{array}$ & Score \\
\hline Type 1 & 3 & 4 & 2 & $\mathbf{9}$ \\
\hline Type 2 & 1 & 4 & 2 & $\mathbf{7}$ \\
\hline Type 3 & 3 & 2 & 1 & $\mathbf{6}$ \\
\hline Type 4 & 0 & 4 & 0 & $\mathbf{4}$ \\
\hline Type 5 & 4 & 2 & 1 & $\mathbf{7}$ \\
\hline Type 6 & 2 & 3 & 2 & $\mathbf{7}$ \\
\hline Type 7 & 4 & 1 & 1 & $\mathbf{6}$ \\
\hline Type 8 & 4 & 2 & 1 & $\mathbf{7}$ \\
\hline
\end{tabular}

\section{TEST DATA}

Following the review of COTS cells, testing must be carried out on the short-listed cells in order to ascertain 
whether they are suitable for typical space conditions.

The initial stage is restricted to those tests that provide go/no-go information, i.e. if the cell cannot survive vacuum, it will be unsuitable for space. In addition, long term tests will be started, so that useful data can be obtained as early as possible. The selected tests are shown in Figure 1.

To date, testing has been started for four of the eight cell types (1-4).

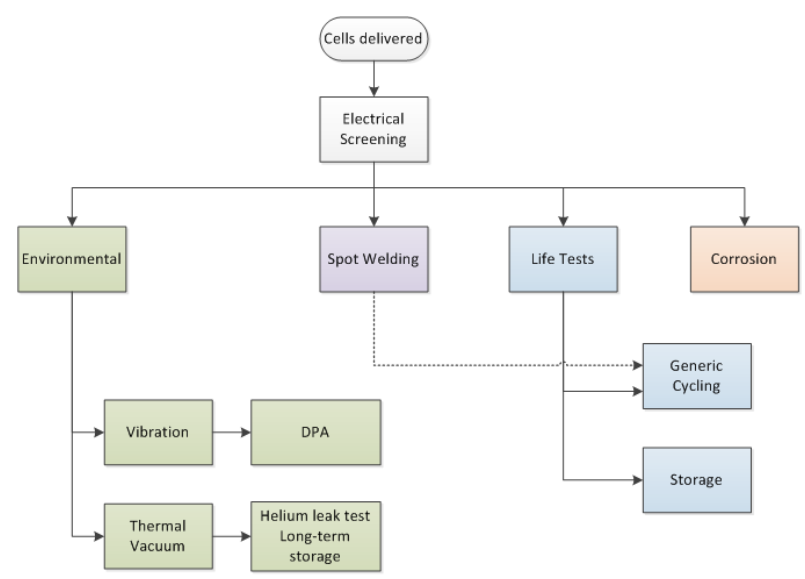

Figure 1. Technology watch test overview

\subsection{Electrical Screening}

The first test any cells undergo is an electrical cycle to determine the capacity and resistance. As ABSL manufacture batteries without balancing and bypass electronics, the cells are closely matched to ensure they stay appropriately balanced throughout the life of the battery. Therefore, cell types with a wide capacity or resistance spread may be more difficult to use. The level of cell rejection is also an important parameter, as it gives an indication of the manufacturing quality.

The normalised capacity results from four cell types are shown in Figure 2. The screening data have been referenced to the manufacturers' 'nominal' capacity from the cell datasheets; the conditions for these values are different between manufacturers and cell types. The screening at ABSL has been carried out using the same equivalent regime (i.e. same $\mathrm{C}$ rates) for all cell types, to provide a fair comparison. The data show that for three of the four cell types, the returned capacity is in line with the manufacturers' nominal value. However, Cell Type 3 returned more capacity than expected.

Aside from the actual capacity, the spread of values was reviewed. Cell Type 3 can be seen to have a narrower distribution than the other cells, which may mean a higher utilisation could be achieved. However, these results are from batches of less than 300 cells, so care should be taken when considering scaling to a full batch.

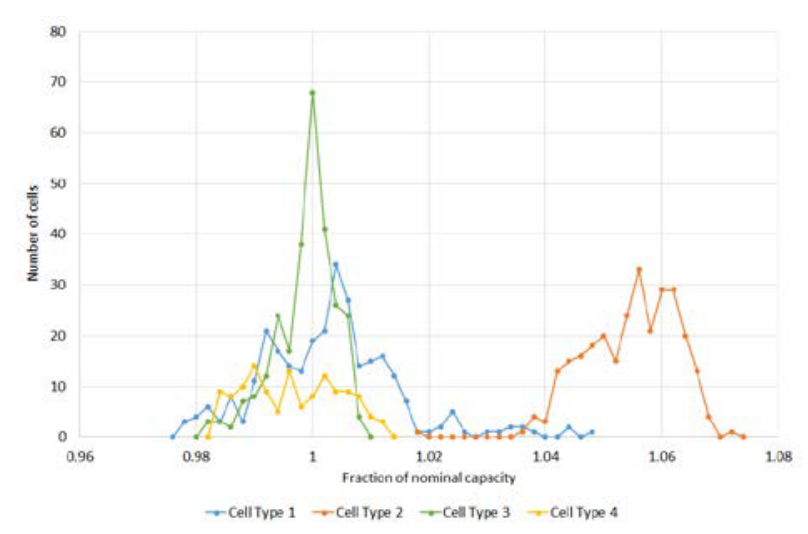

Figure 2. Normalised screening capacity

Another important factor is the cell resistance. Data at end of charge (EoC) is shown in Figure 3. The resistance is dependent on the design purpose of the cells; those designed for high rate applications have lower resistance (for example Cell Types 1 and 2).

As for the capacity, the spread of EoC resistance is important, due to the effect on the level of balancing that can be achieved. Three of the four cell types show low levels of variation in the resistance, however Cell Type 4 shows a much higher level of $40 \mathrm{mOhm}$ out of an average of $180 \mathrm{mOhm}(22 \%)$.

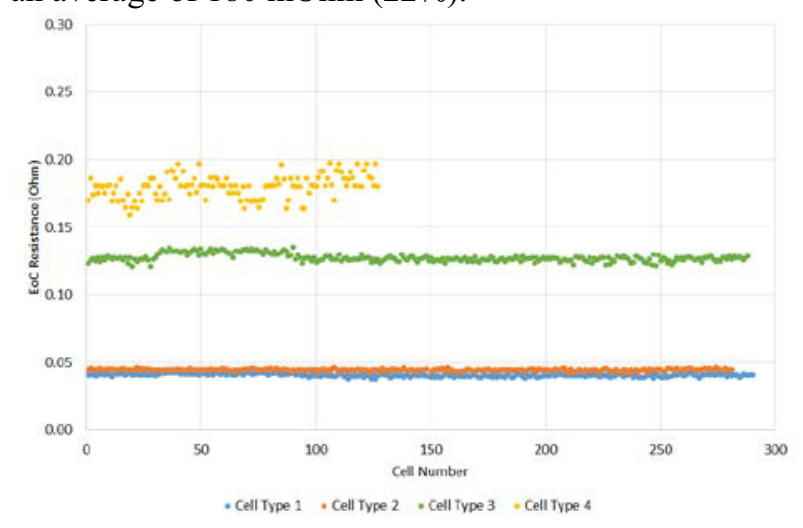

Figure 3. Cell EoC resistance (screening)

The reject rates for the four screened cell types are shown in Table 3. These cells have only been rejected due to electrical parameters being out of family; rejections due to dents, scratches are not taken into account. Three of the cell types have very low reject rates. However, one cell type had a high rate; most of these cells were rejected due to low delivery voltage, which may indicate a high level of self-discharge. 
Table 3. Screening electrical rejection rate

\begin{tabular}{|c|c|}
\hline Cell Type & Reject Rate \\
\hline Type 1 & $1 / 290(0.3 \%)$ \\
\hline Type 2 & $2 / 281(0.7 \%)$ \\
\hline Type 3 & $2 / 290(0.6 \%)$ \\
\hline Type 4 & $15 / 127(12 \%)$ \\
\hline
\end{tabular}

\subsection{Environmental}

To survival of the cells in the space environment is extremely important. Therefore, the performance of the cells will be assessed under typical vibration conditions encountered when an ABSL battery design is subjected to its specified random and sine vibration. The cells must also be able to survive sustained periods at hard vacuum without failure of hermeticity.

In addition to the space environment, batteries and cells are stored on ground prior to launch. Under these circumstances, the corrosion resilience of the cells needs to be evaluated.

To date, the robustness of two of the cell types to vibration has been tested. Figure 4 shows the vibration environment the cells were subjected to. Following the testing, the cells underwent a destructive parts analysis (DPA). The cells were inspected to ensure no signs of damage, for example cracks, tears, or delamination. No differences were found between non-vibrated and vibrated cells, demonstrating their ability to withstand severe mechanical testing.

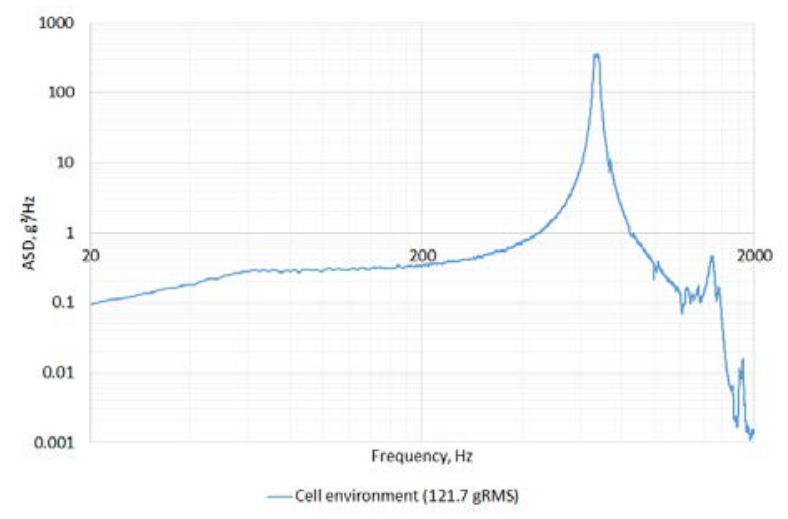

Figure 4. Random vibration test condition

With respect to vacuum and corrosion testing, no data are currently available.

\subsection{Life Testing}

Lifetime performance was identified as an important factor in selecting another cell for space qualification. These tests need to be started as early as possible, due to the long timescales needed to acquire sufficient data for comparison and battery sizing.
For general applications, it is desirable for the cells to show:

- Good consistency within a batch to allow cells to remain balanced through life.

- Low variability within batches to ensure fade/resistance prediction tools do not have to be batch specific.

- Lower fade than the current ABSL cells.

The cell performance will be characterised under regimes typical for space applications. Therefore, the life tests will be conducted at typical LEO conditions (20\% DoD), as well as higher DoD (40\%), which is similar to the average DoD during GEO for ABSL batteries. At present, only the $40 \% \mathrm{DoD}$ tests have been started. Cell tests to determine the fade under storage regimes are also being started.

\subsubsection{Cycling}

The end of discharge (EoD) voltages for three cell types cycled at $40 \%$ DoD are shown in Figure 5. To date, all cell types show a lower rate of decrease in their EoD voltage compared to the heritage HCM cell, as well as the newer NL cell. Therefore, these three cell types currently look promising in terms of long term cycling performance. It should be noted that the HCM cell was cycled at a higher current $(2 \mathrm{C} / 3)$, which explains why the EoD voltage is seen to be lower than for the tests cycled at $\mathrm{C} / 2$.

From previous test experience, the relative behaviour of cells at $40 \%$ DoD has corresponded well with their performance at lower DoDs. It is therefore expected that the cell performance at lower DoDs will also show an improvement over the current ABSL cells. This will be subject to test.

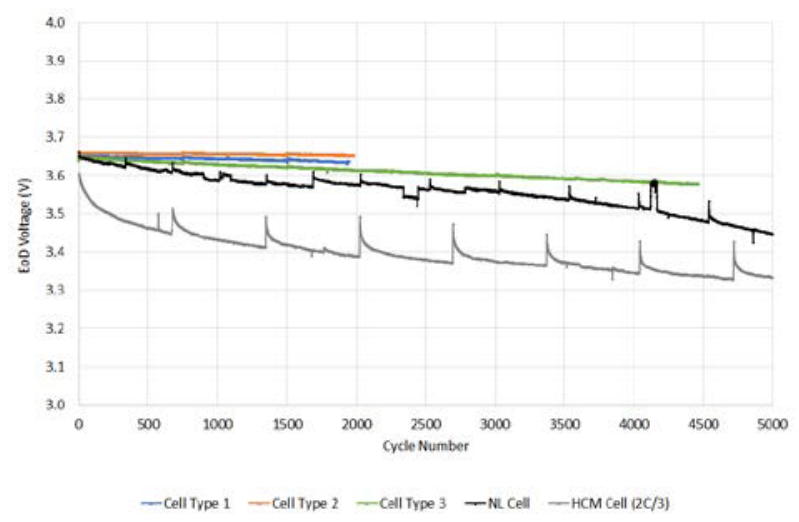

Figure 5. Cycling data $\left(40 \% \mathrm{DoD}, 4.1 \mathrm{~V}, 20^{\circ} \mathrm{C}, \mathrm{C} / 2\right.$ except where stated)

As well as the relative performance of the selected cell types against the HCM and NL cells, the variation within a batch and batch-to-batch needs to be 
determined. If the variation between batches is large, a generalised fade tool is not feasible, and any battery sizing should be conducted using specific batch data (or the worst case). Whereas, variation within a batch may mean the cells would not remain balanced. For example, data for three tests at $40 \%$ DoD on Cell Type 2 are shown in Figure 6. The testing on Batch 1 and Batch 2 were carried out at separate ABSL sites using different batches. These early data show that the results are extremely consistent within a batch, and between batches. These tests will continue to be monitored to determine whether the trends are maintained in the long term.

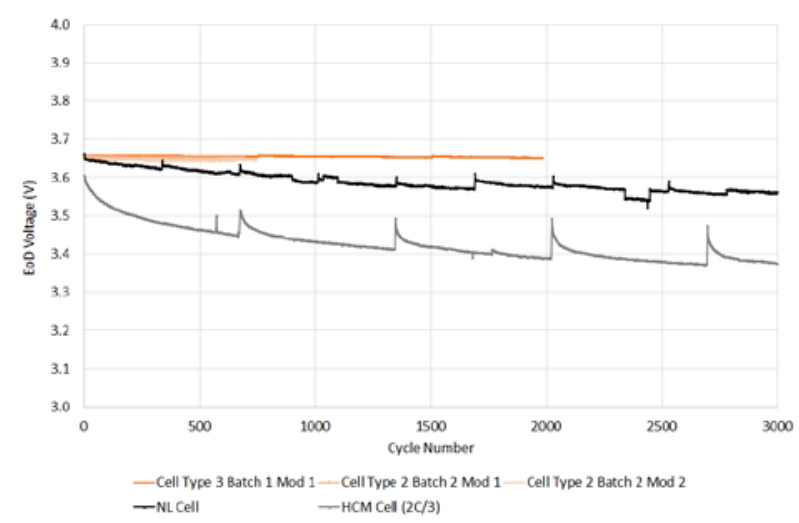

Figure 6. Cycling data showing in batch, and batch-tobatch variation $\left(40 \% \mathrm{DoD}, 4.1 \mathrm{~V}, 20^{\circ} \mathrm{C}, \mathrm{C} / 2\right)$

\subsubsection{Storage}

Most applications will require cells to be stored for a certain period, for example ground storage prior to integration, during a cruise phase, or on a launch pad. $100 \%$ state of charge (SoC) is the worst case and used prior to launch or during satellite test campaigns, whereas long term storage is likely to be between $50 \%$ and $5 \%$ SoC depending on the need for emergency power.

At present, data for storage are only available for one cell type: Cell Type 2. A comparison of the retained capacity against ABSL's current cells, as well as the effect of SoC, are shown in Figure 7. As expected, the higher the $\mathrm{SoC}$, the higher the rate of fade. In terms of the comparison to the HCM and NL, this cell type is showing performance that is at least as good. Therefore, the very early data is promising.

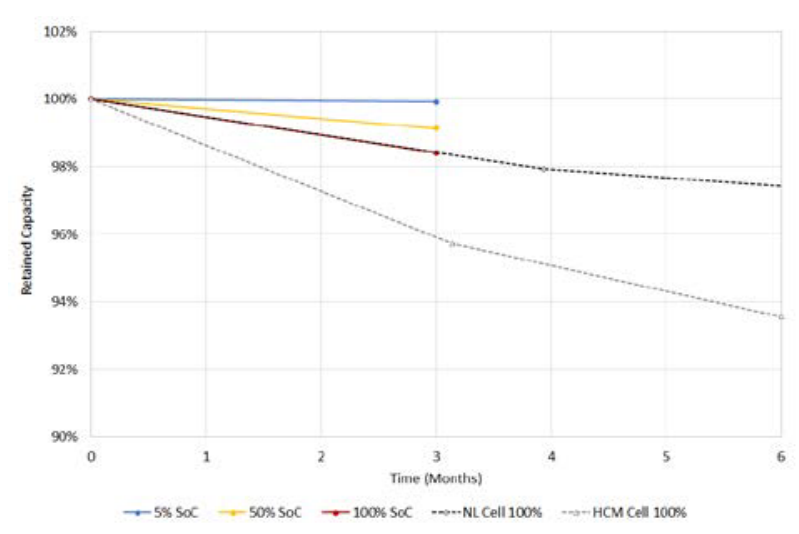

Figure 7. Retained capacity of Cell Type 2 during storage at $20^{\circ} \mathrm{C}$

\section{CONCLUSION}

A review of COTS Li-ion cells has shown that multiple types are available that have higher energy density, longer cycle life, and/or better rate capability than the current ABSL range.

Testing has been started on four or the eight selected cell types. Initial data show that there are some promising candidates for further investigation. Results from cell types with less fade than the HCM and NL cells, as well as low variability, have been presented.

The COTS cell review will continue to amass data on available cells. Four further cell types are about to undergo electrical screening, and additional life tests are due to be started to demonstrate the performance at lower DoD. The environmental testing will continue once all cell types have been screened.

\section{REFERENCES}

1. Power Sources Database, Schmuel De-Leon Energy Ltd.

2. Ligneel, E., Defer, M., Fischer, F., Dumont, E., Fusalba, F., Chami, M., and Gineste, V.; High specific Li-ion cells based on high voltage materials; Executive Summary; ESA Contract 4000107101/12/NL/LvH; 2015. 